\title{
Phakic IOLs: Sulcus Versus Iris-Claw Versus Angle-Supported: Complications and Outcomes
}

\author{
Michael W. Raciti · Parag A. Majmudar
}

Published online: 30 January 2013

(c) Springer Science + Business Media New York 2013

\begin{abstract}
Patient interest in refractive eye surgery is increasing, especially for correction of high levels of myopia and hyperopia. Outcomes of techniques that work well for correcting lower levels of error, such as excimer laser correction, fall short of patient expectations. These expectations have driven the development of new technology for correcting high refractive errors. A new technique, phakic intraocular lens (pIOL) insertion has proven to be safe and effective for treating high myopia and high hyperopia. In this article, the different types of pIOLs are discussed along with recently published results and complications of each design. Larger studies with long term follow up are needed.
\end{abstract}

Keywords Phakic intraocular lens - High myopia . Refractive surgery

\section{Introduction}

The correction of high myopia may present multiple management challenges for the ophthalmologist. At this level of correction, spectacles are less-than-ideal, as they may cause glare, aberrations, and restricted visual field. Contact lenses

M. W. Raciti

Rush University Medical Center, 1725 W Harrison Street, Suite 906, Chicago, IL 60612, USA

e-mail: michael_raciti@rush.edu

P. A. Majmudar $(\square)$

Chicago Cornea Consultants, Chicago, IL, USA

e-mail: pamajmudar@chicagocornea.com

P. A. Majmudar

Rush University Medical Center, 1725 W. Harrison Street,

Suite 928, Chicago, IL 60612, USA are often the non-surgical mainstay, but in many cases, they can be difficult to tolerate due to ocular surface disease or unsafe for some patients. Surgical options for correcting high myopia include excimer laser treatment with photorefractive keratectomy (PRK) or laser assisted in situ keratomileusis (LASIK). However, due to the large amount of corneal tissue that would need to be removed, laser vision correction is of limited value in high myopia. Common concerns include less predictability of the refractive outcome, post-operative refractive instability, and the risk of post-operative ectasia.

Non-corneal surgical approaches to high myopia include refractive lens exchange and phakic intraocular lens (pIOL) insertion. Refractive lens exchange involves removing the clear (cataract-free) crystalline lens and inserting a posterior chamber, myopia-correcting IOL in the capsular bag (phacoemulsification). Refractive lens exchange may be a less desirable procedure, especially in younger patients because it results in total loss of accommodation. There may also be an increased risk of retinal detachment with phacoemulsification in highly myopic eyes, although this data originated with extracapsular cataract surgery, and modern, small-incision phacoemulsification may confer less of a risk of retinal detachment.

Phakic intraocular lenses may be a preferred technique in high myopia due to preservation of accommodation. Phakic IOLs are inserted in front of the crystalline lens, either in the anterior chamber or in the posterior chamber ciliary sulcus. Phakic IOLs are capable of correcting high myopia and hyperopia. Toric models of many pIOLs are also available outside the United States to allow for simultaneous correction of astigmatism as well. In addition to retained accommodative ability of the crystalline lens, advantages of pIOLs include preserved corneal architecture, and potential removability and reversibility. Phakic IOLs have been shown to produce better visual outcomes than refractive lens 
exchange [1] and are thought to offer better quality of vision than excimer laser surgery. More than likely this is due to preservation of corneal architecture and also because the refractive correction takes place closer to the nodal point of the eye. Phakic IOLs may also be more attractive than laser refractive procedures for high myopia because of more predictable outcomes and faster recovery.

A recent Cochrane review of three prospective randomized trials comparing excimer laser to pIOL for the correction of moderate to high myopia concluded that pIOLs are safer than excimer laser [2•]. Patients receiving pIOLs had fewer lines lost of best spectacle-corrected visual acuity at 12 months post operatively; however, there was a low risk of developing early cataract. All three of the trials reviewed indicated that patients who underwent excimer laser surgery were more likely to experience glare and halos as compared to those who had phakic IOLs implanted. The same review also reported that phakic IOL patients had better contrast sensitivity than excimer laser correction. In addition, patient satisfaction and preference questionnaires resulted in higher satisfaction scores in those who had undergone phakic IOL when compared to excimer laser surgery. Similarly, Shin et al. [3] found that posterior chamber pIOL implantation induced fewer ocular and corneal higher order aberrations and resulted in better contrast sensitivity than wave front-guided laser epithelial keratomileusis (LASEK). Recently, Albarrán-Diego et al. [4] published data comparing a foldable iris-fixated pIOL to femtosecond laser-assisted LASIK for myopia -6.00 to -9.00 D. Results showed no difference in spherical equivalent or uncorrected distance visual acuity. However, there was significantly better corrected distance visual acuity and contrast sensitivity in eyes treated with pIOL compared to femtosecond laser-assisted LASIK.

Risks and complications of pIOL insertion are specific to pIOL type and the location of implantation in the eye. Specific complications are discussed in more detail below under each of the pIOL types. In general, risks include endophthalmitis, corneal endothelial decompensation, glaucoma, iritis, and cataract formation. The risk of retinal detachment (RD) is of particular relevance to patients with high myopia. Jiang et al. [5] report on the incidence of RD in highly myopic patients after implantation of pIOLs. The study included 530 highly myopic eyes and they found an overall incidence of RD of $1.5 \%$, which does not differ significantly from the natural history of RD in highly myopic eyes. Therefore, according to this study pIOL insertion does not seem to increase the risk of RD.

To date, only two pIOLs are available for implantation in the United States. Other models are being evaluated by the United States Food and Drug Administration. The goal of this article is to review current literature regarding the complications and outcomes of the three different types of
pIOLs. Recent literature directly comparing different pIOLs will also be discussed below.

\section{Angle Supported Anterior Chamber Phakic IOLs}

Angle supported pIOLs are placed in the anterior chamber and stabilized with haptics that extend into the anterior chamber angle. Currently, there are no angle supported pIOLs that have been approved in the USA. There are two designs available internationally.

The AcrySof Cachet (Alcon, Fort Worth, TX, USA) is a single-piece hydrophobic acrylic IOL, which is currently in FDA clinical trials. The Acrysof has a $6 \mathrm{~mm}$ optic and haptic length ranging from 12.5 to $14.0 \mathrm{~mm}$ sized to accommodate anterior chambers of varying diameter. Recent studies have reported good outcomes with the Acrysof pIOL. One study by Mastropasqua et al. [6] evaluated the lens in 36 eyes with follow up of 1 year and demonstrated good visual outcomes without modification of wavefront refractive errors. Another study by Toso and Morselli [7] evaluated spherical aberrations in 35 eyes pre- and post Acrysof implantation and found improved quality of vision and decreased spherical aberration post-operatively. A smaller study by Yang and Zhao [8] of 25 eyes 1 year after pIOL implantation also supports safety, efficacy, and predictability of the Acrysof pIOL for high to extremely high myopia.

The Kelman Duet (Tekia, Inc.) is a 2 piece design made of poly methyl methacrylate (PMMA). The haptic portion is inserted into the eye followed by the optic. The two pieces are assembled in the anterior chamber. The advantage of this design is that each piece can be inserted through a small $2.5 \mathrm{~mm}$ incision. Also, the optic or haptic can be independently exchanged in the event of power or anterior chamber size calculation errors.

Potential complications of angle supported pIOLs relate to the position of the lens in the anterior chamber angle and the proximity of the lens to the corneal endothelium. Corneal endothelial cell loss is the most common reason for anterior chamber pIOL explantation. Loss for various anterior chamber angle-supported pIOLs ranges from 3.8 to $12 \%$ in the immediate 2 years post implantation. Endothelial cell loss rates many years following implantation range from 0.5 to $1.8 \%$ per year [9•]. Pupil ovalization is another potential complication, which results from deformation of the anterior chamber architecture by the pIOL haptics. Other possible complications include glare and halos, pigment dispersion, pupil block glaucoma, and chronic uveitis.

\section{Iris-Claw Fixated Anterior Chamber Phakic IOLs}

Iris-claw pIOLs are placed in the anterior chamber and fixated to the anterior surface of the iris by a process known 
as enclavation. One model is available in the US, the Verisyse phakic IOL (Abbott Medical Optics, Inc., Santa Ana, CA, USA). This is a single piece non-foldable IOL made of Perspex CQ-UV, a UV-filtering PMMA material [10]. The same iris-claw model has been available in other countries for decades and is known there as the Artisan lens (Ophtec BV). There are two optic sizes (5.0 and $6.0 \mathrm{~mm}$ ) available to correct high myopia and high hyperopia. In Europe, additional iris-claw pIOLs are available, as well a toric phakic IOL model and a foldable phakic IOL model [Artiflex (Ophtec BV)] which has the advantage of being able to be implanted via a $3.2 \mathrm{~mm}$ incision, compared to the $6.5 \mathrm{~mm}$ incision required by the Verisyse or Artisan lens. The name given to the sister model of the Artiflex in the United States is the Veriflex (Abbott Medical Optics) and this lens model is currently undergoing clinical trials in the USA.

A recent study evaluated the stability and predictability of visual outcomes 6 months after insertion of the Artiflex pIOL and showed favorable results [11]. Two other studies with 5 year follow up following implantation of the Verisyse pIOL have also recently been published. In one study, 85 eyes were evaluated and the Verisyse pIOL demonstrated effective, predictable, and stable outcomes [12]. In the other study, 84 eyes were evaluated and there was a significant reduction in spherical error, with no loss of best spectacle corrected visual acuity [13]. Munoz et al. [14] report similarly effective results with the foldable toric model pIOL, the Artiflex toric (Ophtec BV).

The main advantages of iris-fixated lenses include the fact that they can be more easily centered over the pupil and there is no need to measure anterior chamber or ciliary sulcus diameter. The larger $6.0 \mathrm{~mm}$ optic size of the Artisian lens seems to induce less glare and halos from presumed edge effect [9॰].

Iris-claw fixated pIOLs are especially attractive as toric models because they are more rotationally secure in the anterior chamber are than haptic supported pIOLs. However, a toric sulcus pIOL produced significantly better uncorrected visual acuity than an iris-fixated pIOL in a recent study, which is discussed below in the comparison section [15].

Like angle supported pIOLs, iris-claw pIOLs are implanted in the anterior chamber and therefore also pose a similar risk of corneal endothelial cell decompensation. Reports of endothelial cell loss are highly variable in different studies. Three studies report no change at 6 months to 2 years. Others report anywhere from $0.7 \%$ to as high as $17.6 \%$ at 24 months [9॰]. Kohnen et al. maintain that patients with endothelial cell counts below $2,000 / \mathrm{mm}^{2}$ should not receive a pIOL.

Since iris-claw pIOLs are fixated directly to the iris, pigment dispersion and chronic uveitis are a possible concern. However, rates of these complications have been low [9•]. Similarly, the incidence of pupil distortion, elevated intraocular pressure and cataract formation following iris-fixated pIOL are also very low.

\section{Sulcus Phakic IOLs}

Posterior chamber pIOLs are placed in the ciliary sulcus with haptics supporting the central optic. Pre-operative measurements must determine the diameter of the sulcus so that the haptics securely support the IOL without placing undue pressure in the sulcus, or creating iris chafing.

One model is FDA-approved and available in the United States, the Visian ICL (Implantable Collamer Lens) (Staar Surgical Co., Monrovia, CA, USA). The lens is comprised of collamer $(0.2 \%$ collagen and $60 \%$ hydroxyethyl methacrylate copolymer), which is a proprietary hydrophilic collagen copolymer with UV absorbing properties [16]. Another sulcus pIOL, not available in the USA, is the Phakic Refractive Lens (PRL; Carl Zeiss Meditec Inc., Jena, Germany), which is a thin sulcus pIOL made of silicone. A recent study with follow up as long as 6 years shows PRL implantation is an effective and safe for treating high myopia [17].

Sulcus pIOLs have less risk of corneal endothelial compromise because they are further from the cornea. They are thought to have a lower risk of halos and glare since the margins of the pupil cover the border of the lens. However, patients with large scotopic pupils may experience halos and glare if the pupil dilates beyond the edge of the optic. Therefore, mesopic pupils larger than $5 \mathrm{~mm}$ are a relative contraindication for these lenses.

With sulcus-based pIOLs, there is an inherently higher risk of cataract formation given its close proximity to the crystalline lens. Hyperopia-correcting models, which are thicker centrally, are especially prone to causing cataract since they may not adequately vault over the anterior surface of the lens. The distance between a posterior chamber pIOL and the lens also appears to be dynamic, and therefore even a properly sized pIOL may at times come into contact with the crystalline lens, or at the very least alter the fluid dynamics of the aqueous in front of the crystalline lens, and therefor predispose the patient to early cataract formation. Evidence for this is provided through ultrasound biomicroscopy, which reveals that the distance between the anterior crystalline lens surface and the Visian ICL is reduced during pharmacologic accommodation [18].

The most common type of cataract induced is the anterior subcapsular cataract. A meta-analysis of cataract development after posterior chamber pIOL surgery found an overall incidence of cataract formation to be $9.60 \%$ [9॰]. This percentage included the Adatomed pIOL with a cataract incidence of $25.7 \%$; however this lens in no longer 
available in Europe. According to Moshirfar et al., anterior subcapsular cataracts are thought to result acutely from operative trauma and include vacuoles and flecks, which are usually not visually significant. On the other hand, anterior subcapsular cataracts that occur years after pIOL insertion are often progressive and decrease vision. Late cataracts are hypothesized to result from chronically inadequate aqueous perfusion or chronic inflammation [19].

Sulcus pIOLs may also be positioned too anteriorly and may cause pupillary block glaucoma or iris chafing and pigment dispersion. Anterior segment imaging techniques such as anterior segment OCT (Visante OCT, Carl Zeiss Meditec Inc.) and ultrasound biomicroscopy allow for more accurate sizing of sulcus pIOLs and are likely to decrease complication rates with these lenses [20]. Sulcus pIOLs have been reported to dislocate into the anterior chamber following trauma and can be safely repositioned in the posterior chamber [21]. Sulcus-based lenses may also migrate posteriorly into the vitreous cavity through eroded zonules. Other risks include chronic iritis, pupillary block, and pigment dispersion glaucoma if the lens vaults inappropriately anteriorly, but these complications are rare.

\section{Phakic IOL Comparison}

Data comparing efficacy and safety of different models and designs of pIOLs are beginning to emerge. Hassaballa et al. [22] showed that the Visian ICL sulcus pIOL and Artisan iris-claw pIOL display equal and comparable safety, predictability, and efficacy. Lee et al. [15] compared visual outcomes in patients receiving one of two toric pIOLs, the Visian toric ICL or the Artisan toric pIOL. They found similar correction of refractive error with both lenses, but $\operatorname{logMAR}$ uncorrected visual acuity was significantly higher in the Visian toric ICL group at 1, 3, and 6 months postoperatively. Differences are thought to be due to different amounts and locations of surgically induced astigmatism. Best spectacle corrected visual acuity was not significantly different between the two lenses.

\section{Conclusion}

In conclusion, pIOL implantation is a safe option for treating moderate to high myopia and hyperopia. In fact, recent data suggests pIOL insertion may be the best option for treating high ametropia because pIOLs appear to be more effective at maintaining high visual quality compared to other treatments such as excimer laser correction, which permanently alter the corneal architecture. As newer pIOL models are approved in the United States, there will be greater options for surgeons to evaluate. As the safety and efficacy of pIOL implantation increases, these lenses may be thought of as more of a firstline treatment even for lower degrees of ametropia.

Disclosure None.

\section{References}

Papers of particular interest, published recently, have been highlighted as:

- Of importance

1. Nanavaty MA, Daya SM. Refractive lens exchange versus phakic intraocular lenses. Curr Opin Ophthalmol. 2012;23(1):54-61.

2. Barsam A, Allan BD. Excimer laser refractive surgery versus phakic intraocular lenses for the correction of moderate to high myopia. Cochrane Database Syst Rev. 2012;1:CD007679. This publication is a current review comparing a widely accepted technique for refractive surgery (excimer laser) to an emerging technique (phakic IOL insertion).

3. Shin JY, Ahn H, Seo KY, Kim EK, Kim TI. Comparison of higher order aberrations after implantable Collamer Lens implantation and wavefront-guided LASEK in high myopia. J Refract Surg. 2012;28(2):106-11.

4. Albarrán-Diego C, Muñoz G, Ferrer-Blasco T, García-Lázaro S, Belda-Salmerón L. Foldable iris-fixated phakic intraocular lens vs femtosecond laser-assisted LASIK for myopia between -6.00 and -9.00 diopters. J Refract Surg. 2012;28(6):380-6.

5. Jiang T, Chang Q, Wang X, Huang X. Retinal detachment after phakic intraocular lens implantation in severe myopic eyes. Graefes Arch Clin Exp Ophthalmol. 2012;250(12):1725-30.

6. Mastropasqua L, Toto L, Vecchiarino L, Doronzo E, Mastropasqua R, Di Nicola M. AcrySof cachet phakic intraocular lens in myopic patients: visual performance, wavefront error, and lens position. J Refract Surg. 2012;28(4):267-74.

7. Toso A, Morselli S. Visual and aberrometric outcomes in eyes with an angle-supported phakic intraocular lens. J Cataract Refract Surg. 2012;38(9):1590-4.

8. Yang RB, Zhao SZ. AcrySof phakic angle-supported intraocular lens for the correction of high to extremely high myopia: oneyear follow-up results. Int J Ophthalmol. 2012;5(3):360-5.

9. - Kohnen T, Kook D, Morral M, Güell JL. Phakic intraocular lenses: part 2: results and complications. J Cataract Refract Surg. 2010;36(12):2168-94. This is an excellent review of current results and complications of phakic IOLs currently in use.

10. Güell JL, Morral M, Kook D, Kohnen T. Phakic intraocular lenses part 1: historical overview, current models, selection criteria, and surgical techniques. J Cataract Refract Surg. 2010; 36(11):1976-93.

11. Doors M, Budo CJ, Christiaans BJ, Luger M, Marinho AA, Dick HB, Güell JL, Nuijts RM. Artiflex toric foldable phakic intraocular lens: short-term results of a prospective European multicenter study. Am J Ophthalmol. 2012;154(4):730-9.

12. Titiyal JS, Sharma N, Mannan R, Pruthi A, Vajpayee RB. Irisfixated intraocular lens implantation to correct moderate to high myopia in Asian-Indian eyes: five-year results. J Cataract Refract Surg. 2012;38(8):1446-52.

13. Yuan X, Ping HZ, Hong WC, Yin D, Ting Z. Five-year follow-up after anterior iris-fixated intraocular lens implantation in phakic eyes to correct high myopia. Eye. 2012;26(2):321-6.

14. Muñoz G, Cardoner A, Albarrán-Diego C, Ferrer-Blasco T, BeldaSalmerón L. Iris-fixated toric phakic intraocular lens for myopic astigmatism. J Cataract Refract Surg. 2012;38(7):1166-75. 
15. Lee SY, Kwon HJ, Ahn HS, Seo KY, Kim EK, Kim TI. Comparison of patient outcomes after implantation of Visian toric implantable collamer lens and iris-fixated toric phakic intraocular lens. Eye. 2011;25(11):1409-17.

16. Lovisolo CF, Reinstein DZ. Phakic intraocular lenses. Surv Ophthalmol. 2005;50(6):549-87.

17. Portaliou DM, Kymionis GD, Panagopoulou SI, Kalyvianaki MI, Grentzelos MA, Pallikaris IG. Long-term results of phakic refractive lens implantation in eyes with high myopia. J Refract Surg. 2011;27(11):787-91. doi:10.3928/1081597X-20110628-01.

18. Du C, Wang J, Wang X, Dong Y, Gu Y, Shen Y. Ultrasound biomicroscopy of anterior segment accommodative changes with posterior chamber phakic intraocular lens in high myopia. Ophthalmology. 2012;119(1):99-105.
19. Moshirfar M, Mifflin M, Wong G, Chang JC. Cataract surgery following phakic intraocular lens implantation. Curr Opin Ophthalmol. 2010;21(1):39-44.

20. Güell JL, Morral M, Kook D, Kohnen T. Phakic intraocular lenses part 1: historical overview, current models, selection criteria, and surgical techniques. J Cataract Refract Surg. 2010;36(11): 1976-93.

21. Schmitz JW, McEwan GC, Hofmeister EM. Delayed presentation of traumatic dislocation of a Visian Implantable Collamer Lens. J Refract Surg. 2012;28(5):365.

22. Hassaballa MA, Macky TA. Phakic intraocular lenses outcomes and complications: Artisan vs Visian ICL. Eye. 2011;25(10): 1365-70. 